\title{
Integration of Satellites into GSM: Signaling Flow Analysis
}

\author{
F.Argenti, L.Cappelletti, E.Del Re, A.Ferro \\ Dipartimento di Ingegneria Elettronica, Università di Firenze \\ Via di Santa Marta, 3 - 50139 Firenze
}

\begin{abstract}
In this work the signaling flow necessary for the integration between the paneuropean digital mobile network GSM and a satellite component is discussed. The problem of integrating GSM with satellites is of great importance since it can be considered as a first step towards the definition of the Universal Mobile Telecommunications System (UMTS), where satellites play a fundamental role in obtaining a global coverage communication network.
\end{abstract}

\section{Introduction}

Terrestrial mobile networks, like GSM, provide communication services to a growing number of mobile users. However, in some regions, for example sparsely inhabited areas, the deployment of GSM infrastructures may be not convenient and the use of satellites can be economically more attractive. Thus the problem of interworking between the GSM and a satellite component arises. Global coverage is one of the main objectives of the UMTS, the third generation of mobile networks. UMTS is a completely new network and now is in the standardization stage. Since UMTS is not already available and due to the amount of investments spent in the design and standardization of the GSM network, it would be desirable that part of these structures were utilized in a global coverage network based on GSM. Therefore, the problem of the integration of a satellite component into GSM can be seen also as a first step of the migration from second to third generation mobile networks.

To deal with this problem, several projects have been set-up: among these, the European Union funded RACE project "SAINT", whose main objective was the definition of a satellite component for UMTS and, secondarily, the integration of satellites into GSM [1].

\section{Architecture and general assumptions}

A first step in the study of the integration of satellites into GSM is the definition of a possible architecture for the integrated system. GSM is already defined as an independent network; changes to the architecture and protocols of the GSM are assumed not acceptable. Therefore, the satellite component must be seen as a network that is superimposed to the terrestrial one: integration means that part of the terrestrial network elements and procedures must be reused. The higher level of integration is achieved, the higher degree of reuse of terrestrial components, both hardware and software, is possible.

A possible choice of the integrated architecture foresees a reuse of GSM architecture up to the switching elements (Mobile Switching Centers, MSC), as shown in Fig. 1. This means that the databases of the GSM (the HLR and the VLR) and the MSC

0-7803-3300-4/96\$5.00@1996 structure must not be changed, as well as all the interfaces between these network elements.

In this integration scenario, the Fixed Earth Station (FES) that provides the radio link with the satellite and, therefore, with the user, can be seen as the satellite segment counterpart of the terrestrial BSS (BSC+BTSs) ${ }^{1}$; therefore, the MSC must communicate with this entity as it were a BSS, i.e., no changes are allowed to the A interface of the GSM.

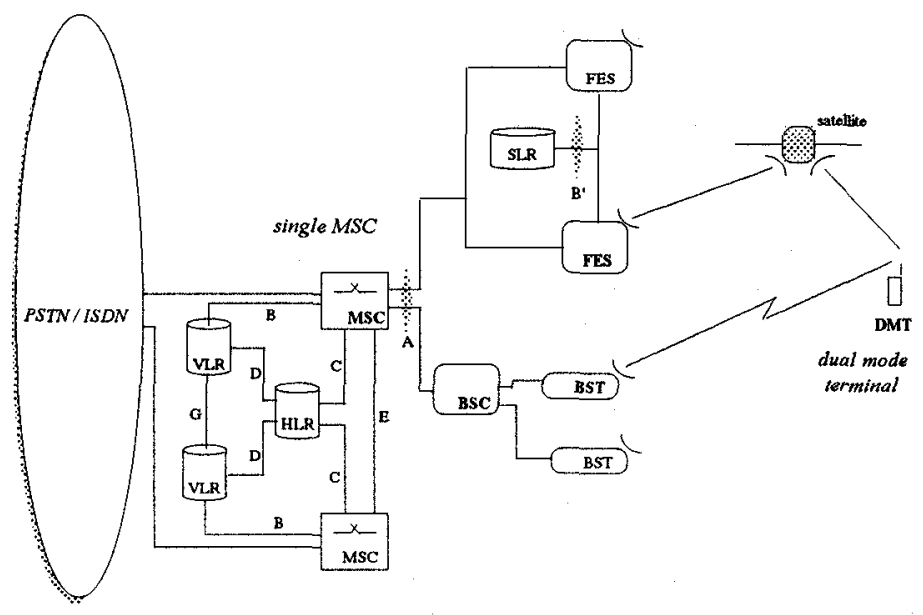

Figure 1 - Satellite-GSM integrated system scenario.

In this work, changes are considered admissible only at the handheld dual mode terminal (Mobile Station, MS) site as well as in the satellite segment components and radio interface. As to the type of satellite component, a Low Earth Orbit (LEO) constellation of satellites will be considered. Work on the integration of a GEO satellite component into GSM was done, for example, in [2].

\section{Mobile users localization}

One of the main problems to be dealt with when the procedures of the GSM are mapped onto the satellite component is that of locating mobile users on the earth surface. In GSM the Location Area (LA) where a mobile user can roam in without updating its position is fixed and can be associated to a set of Base Stations (BS). LEO satellites are the most suitable for satellite Personal Communications: however, due to their high speed with respect to the earth surface, different localization methods than that adopted in GSM could be taken into account.

1 We will not further detail the FES architecture that will be composed by the satellite counterparts of the terrestrial BSCs and BTSs. 
In [3] the "Satellite-fixed cell coverage" and "Earth-fixed cell coverage" concepts are described: in the first approach, the footprint of a satellite beam is considered as moving on the earth surface, according to the satellite motion, so that location updating do not depend on user mobility; in the latter, as in the terrestrial cellular case, the cell are fixed on the earth surface and beam steering and cell switching are used to enable their continuous coverage. Earth-fixed cells seem facilitate the integration process as the location procedure would be closer to the terrestrial one.

A list of other methods are presented in [4] and the problem has also been discussed in SAINT Project [5][6]. Signaling load due to location updating is considered too heavy in "satellite-fixed cells coverage" [7][8]. This load would be reduced if the mobile user (or the FES) were able to determine its position, measured as coordinates on the earth surface, by using GPS [4] or by using Doppler and propagation delay measured on a signal transmitted by the user [5]: in this case, the user location must be updated only if the difference between two measured positions is greater than a given uncertainty radius.

In [4] methods based on the report of spot beams visible from the mobile user are shown: the Location Area in these cases is the intersection between the spot areas at that time.

Another approach introduces an "earth-fixed area" relative to a given FES, that is the area covered at any time by satellites controlled by that FES [5][6]: the association between Mobile Station (MS) and FES-area is performed by monitoring the $\mathrm{BCCH}$ channels broadcast by satellite spot beams or by a terminal position measurement system, that should be available as in the position based approach.

However, it seems clear that, in the chosen integration scenario, information relative to only the space segment cannot be stored in the GSM databases. Therefore a new database, denoted in Fig. 1 as SLR (Satellite Location Register) is introduced in the satellite segment to store whatever information the satellite operator considers necessary to localize the mobile user: this database should store, for example, mobile coordinates in the position based approach and satellite and spot beam used to contact the FES in the FES-area approach.

To summarize, we will indicate with Satellite Location Area (SLA) the uncertainty area in which the mobile can roam without updating its position and with Satellite Location Information (SLI) the information relative to only the satellite component and not transmitted to the GSM databases:

1) in the "position" based approach SLA is the area centered in the mobile user and with a given uncertainty radius; SLI are the mobile coordinates and uncertainty radius;

2) in approaches based on "spot beam report", the SLA is the intersection between the visible spot beam areas; the SLI is the position of the spot beams at the time of the report;

3 ) in the FES-area approach the SLA coincides with the area covered at any time by a FES; to allow a more efficient paging SLI can be either the coordinates computed by the FES and that allow to assign the mobile to a given FES area or the couple (spot beam, satellite) used to contact the FES.

We will not detail the signaling between MS and FES, since this interface must still be defined. The signaling flow necessary for the procedures of Mobility Management, Paging, Call Set-up and Handover will be analyzed.

\section{Mobility Management procedures}

The procedure of Location Updating permits to store/update in the network the information about mobile user position; the information relevant only to the satellite component (SLI) can be stored into a special database (SLR). Depending on the used localization approach the impact on the signaling flow is different. However, whatever the chosen localization approach, it is clear that the information stored in the VLR associated to the MSC the FES is connected to must have the same format as the GSM Location Area Identifier (LAI), shown in Fig. 2.

MCC $\mathrm{MNC} \quad \mathrm{LAC}$

MCC: Mobile Country Code (3 digit)

MNC: Mobile Network Code (2 digit)

LAC: Location Area Code (2 octets)

Figure 2 - GSM LAI format

In the case of a mobile registered in the satellite environment, the LAC will contain a FES identifier (FESC).

In "satellite-fixed cell coverage" each spot beam is characterized by different $\mathrm{BCCH}$ channels so that, when the mobile detects a stronger signal on a channel different from the selected one then it triggers a location updating procedure. Such approach would lead to a transparent reuse of GSM procedures but it seems to produce a very heavy signaling load, so that we will consider here the other approaches described in Section 3.

\section{I Registration}

When the mobile terminal registers for the first time into the satellite environment, a list of the satellite link frequencies that are visible is compiled and the best received one is used to contact the network.

1) In the "position" based approach, the mobile sends (or lets the FES compute) its position to the network.

2) In the "spot beam report" the mobile communicates the spot beam that are visible.

3) In the "FES-area" approach the mobile communicates the FES area code that is broadcast on the $\mathrm{BCCH}$ channel of the spot that is better received.

In all these approaces, the FES that is contacted stores the mobile SLI in its SLR and sends its own code to the GSM network, that is stored in a GSM VLR in LAI format.

\subsection{Location Update}

Consider the following procedure to update the location of a mobile into the integrated system:

i) the mobile registers, if possible, into the terrestrial segment and performs, when necessary, an update of the location area into the GSM network; 
ii) if a terrestrial link is not or no more available (for example the mobile is leaving of the terrestrial coverage), then it selects a spot beam and registers into the satellite segment; then, when necessary, it performs a location update into the satellite segment, but, periodically, it tries to find terrestrial resources available: if this attempt is successful it changes segment performing a location update.

As can be seen the satellite segment is selected if no terrestrial resources are available; however, if the satellite segment is presently selected, a search for terrestrial channels is periodically attempted. This technique is to save as much as possible satellite channels that are considered more expensive.

Therefore, different types of location updating must be considered: if the mobile is registered into the terrestrial segment, it can select a new terrestrial (this is the GSM procedure) or a satellite LA; if it registered into the satellite segment, then it can select a new satellite LA or a terrestrial cell.

Intra Satellite Segment Location Update: Suppose the mobile is registered into the satellite segment. Then a FES code (FESC), instead of a GSM LAC, is stored in one of the GSM VLRs; the same code is stored in the SIM card of the mobile user.

According to the localization approach, the mobile contacts the network to trigger a location update:

1) In the "position based" approach the mobile detects (or let the FES periodically measure) that its position is outside the uncertainty area known by the network; then, it informs the network about the new situation;

2) In the "spot beam report" approach the mobile periodically communicates the visible spot beams;

3) In the "FES area" approach a location update is initiated when, by decoding the BCCH, a new FES area is detected.

These communications occur by using the better link available: the FES that is contacted can be different than the one contacted the last time. This situation is depicted in Fig. 3, where FESn and SLRn are the entities presently contacted for the location update, FESo and SLRo are the entities contacted at the time of the last location update. If FESn and FESo coincide the signaling simplifies.

The MS sends a LOC.UP.REQ. message: this message has the same GSM format, apart from the fact that the field dedicated to the LAC contains the address code of the FES stored in the MS. The new FES, using the LAI, knows the code of the old FES: if the FES are different, an update of the GSM databases is performed by using the GSM protocols. The new FES transfers the LOC.UP.REQ. message to the new MSC, which, therefore, receives the code of the old FES. The MSC has to update the VLR and the network has to change the routing data in the HLR. The new VLR is also in charge of contacting the old VLR in order to obtain the information useful for the authentication and ciphering.

The network performs also the authentication of the MS and sets the suitable ciphering mode to transfer and to receive information to and from the MS. The procedures are equal to the GSM ones and involve the same type of messages exchanged in the terrestrial environment. It is to be noted that for these procedures, the FES assumes the behaviour of a transparent relay for the link between the MSC and the MS.

After this, the new VLR provides a new TMSI to the MS sending a LOC.UP.ACCEPT message to the FES. The FES receives this message: if the message contains an IMSI, the FES deletes any TMSI in the SLR; if the message contains a (new) TMSI, the FES stores the received TMSI in the SLR. Moreover, the FES is in charge of informing the MS of the new TMSI forwarding the LOC.UP.ACCEPT message. The MS stores the received TMSI and the new FESC, then it sends a TMSI REALLOCATON COMPLETE message to the network; here the FES behaves as a relay. The MSC completes the procedure by transferring a CHANNEL RELEASE message to the FES.

The new FES is in charge of storing the new SLI and deleting the SLI contained in the old SLR by contacting the old FES: to allow this, direct connection between FESs must be provided.

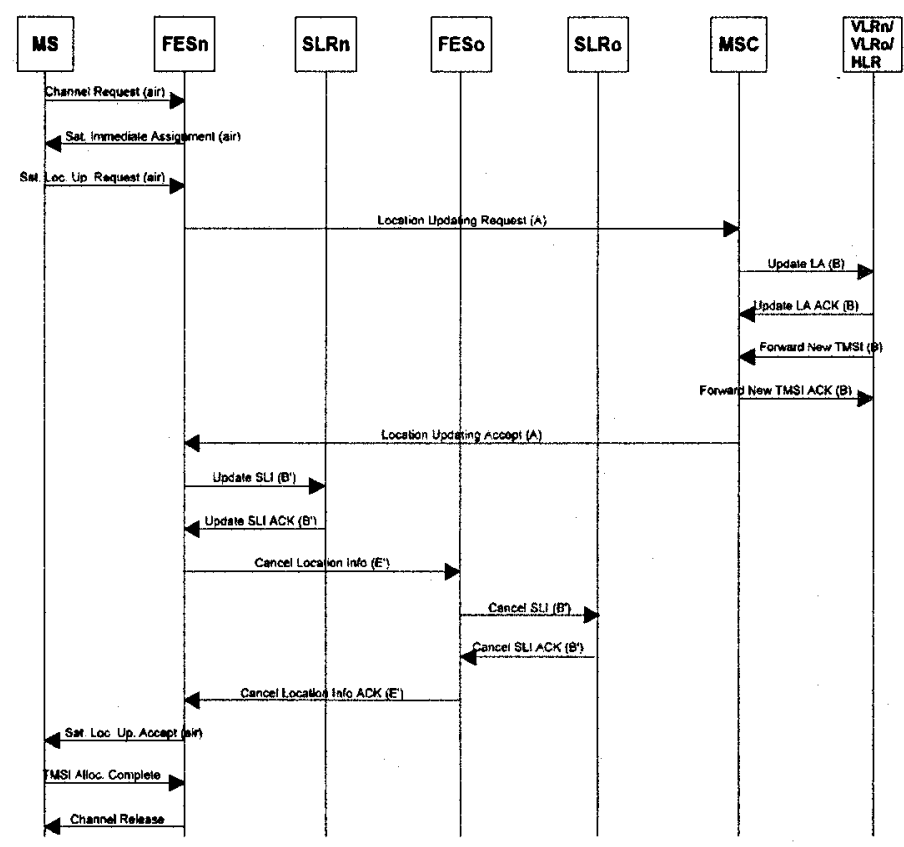

Figure 3 - Signaling flow for Intra Satellite Segment Location Update

GSM to Satellite location update: Suppose now that a MS is registered into a GSM cell and that the MS has a GSM LAI stored into the SIM card. Suppose the MS decides to select a satellite resource and starts a location updating procedure by sending the LOC.UP.REQ. message containing its GSM LAI. The involved FES establishes a connection with its MSC and forwards the LOC.UP.REQ. message containing the GSM LAI. Then the network is able to update the GSM databases as shown before and the FES updates its SLR.

Satellite to GSM location update: When the MS has selected the satellite environment, the MS is registered both in the terrestrial databases (VLR and HLR) and in the satellite one (SLR). The SIM card and the visited VLR store the FESC where the MS is roaming in. As soon as a GSM resource is available, the MS selects a terrestrial $\mathrm{BCCH}$ and compares the received $\mathrm{LAC}$ with 
the stored FESC. A mismatch occurs and the location updating procedure is started by sending a LOC.UP.REQ. message. The location area data field is filled with the FESC, while the contacted BS establishes a connection with the MSC. The network uses the FESC to identify the old VLR and proceeds to move all the MS useful information from the old VLR to the new one. At the end of this data exchange, the MSC updates the VLR number in the HLR. The TMSI allocation follows the same steps than in GSM and the satellite environment is abandoned. Now a problem arises: how does the network inform the FES that all the data related to the MS and still stored in the SLR are no longer useful ? Since the FES is periodically contacted by the MS in order to report its terminal position to the network, the lack of contact could be interpreted by the FES as the abandoning of the satellite environment. This could be revealed by a timer: when it expires, the FES deletes the MS record in the SLR.

\section{Paging and Call Set-Up}

In the "position" and "spot beam report" based approaches considered for the localization in the satellite environment, the SLA may be covered by different spot-beams and, afterwards, by different satellites. The network knows the motion of the satellite constellation and it is always able to choose the most suitable satellite resources to perform an efficient paging in a known terrestrial zone.

Since it is mandatory not to change the GSM entities and their functionalities, the mapping function has to be performed by the FES whose code is stored in the VLR of the GSM network. In GSM, the paging message that comes from the MSC is broadcast through the BTSs of the same LA and, as regards paging, the BS behaves like a relay. In integrated GSM, the paging message arrives at one FES (the only element of the satellite segment known by GSM through its code). This FES manages the paging procedure: if it involves more than one satellite and more than one FES, then it must contact the new FES to allow serial or parallel paging. However, this event, that occurs only when the SLA is at the border between two satellite footprints, seems less likely than the single FES paging.

In Fig. 5 the signaling flow of a mobile destined call is shown. As can be seen, the paging procedure involves differences with the GSM protocols and architecture: in fact, the FES, which is the counterpart of the GSM BSS, now needs to decode the paging message from the GSM network to establish if it is necessary to contact a new FES and, in this case, to allocate traffic resources, and not only signaling resources, with the new FES. This is quite different from the GSM architecture, where no connections exists between BSSs.

\section{Intra-Satellite Segment Handovers}

Different types of handover can be considered in an integrated system: intra segment handovers, that is within terrestrial or satellite segments, and inter segment handover, that is from GSM to satellite segment and viceversa. The intra satellite segment handover can be further divided into intra spot beam, inter spot beam and inter satellite handovers. In this work we will discuss only intra satellite segment handovers, with the call handed over between two different FESs: inter segment handovers seem very difficult to be performed without changes to the GSM protocols.

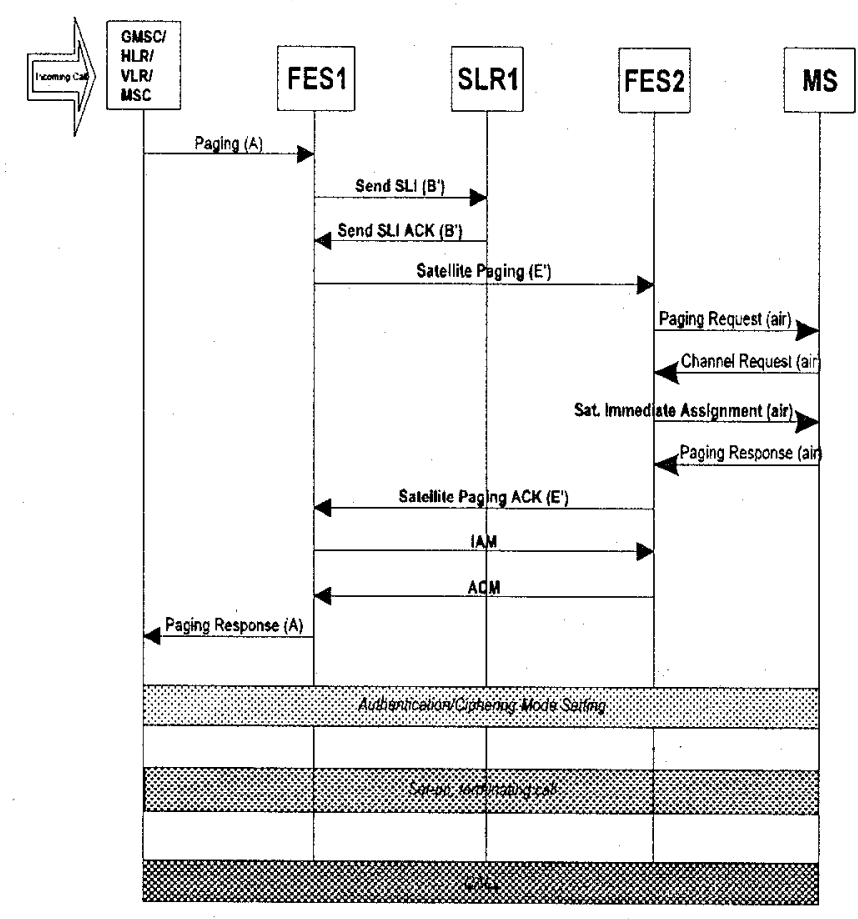

Figure 4 - Signaling flow for Paging into the Satellite Segment

Intra Satellite Segment Handovers take place between two different satellite channels and have the purpose to ensure that the connection to the MS is maintained as it moves from one area to another as well as the satellites of the constellation move around the earth. Because of the high speed, handovers must be performed frequently and rapidly.

Handover decision can be taken only by the network, in particular only by the FESs. GSM MSCs can not be involved in space segment handovers since it would require knowledge of the satellite environment and traffic distribution at the GSM MSC sites. Handover decision will be taken according to a chosen handover strategy based on measurements of radio subsystem performance and signal strength received from both MS and FES.

Handover execution depends on the handover type required at the moment. The substantial difference is between Intra-FES Handover (which takes place between channels controlled by a single FES) and Inter-FES Handover (where the MS is handed over from one FES to another).

Intra-FES Handover can be considered as the space segment counterpart of the GSM internal handover. As the MS is connected with the same FES, no synchronization problems arise, so that the Intra-FES Handover procedure includes only two messages sent on the Air Interface. The FES will send a SATELLITE ASSIGNMENT COMMAND message to the MS, which will describe the assigned channel. The MS will try to hand over the new channel: physical information, like timing retard and Doppler shift, will have also to be provided. In case 
of success, the MS will send back an ASSIGNED COMPLETE message on the new channel, otherwise an ASSIGNMENT FAILURE message will be received by the FES on the old channel.

Inter-FES Handover can instead be considered the space segment counterpart of the GSM external handover. In order to avoid GSM modifications, GSM MSCs will be excluded from this procedure. So Inter-FES handover can be performed only if traffic channels between FESs are provided. In this way, the entire procedure will occur on the Air Interface and on the interface between FESs (that will be named "E' Interface").

The purpose of the signaling flow on the E' Interface is to allow the exchange of information between two FESs (SATELLITE HANDOVER REQUEST and SATELLITE HANDOVER REQUEST ACK messages) and to establish/release a dedicated connection between them (IAM, ACM, SEND END SIGNAL, RELEASE). These messages are usually sent on the $E$ interface between MSCs. Therefore, the FESs assume part of the functionalities that are characteristic of the GSM MSC: this allows to avoid the MSCs to manage satellite resources. In a higher level of integration this should be avoided and radio resources managing should be separated from wired terrestrial resources managing.

The signaling flow on the Air Interface will allow the synchronization of the MS with the new FES. FESs must be synchronized. The change of channel may be performed through a procedure similar to the GSM Handover procedure, which will include at least five messages. If the network knows the MS position, the number of messages exchanged on the Air Interface can be reduced. In fact the new FES can calculate the timing retard and Doppler shift in advance, so that the MS can hand over the new channel at once. In this case only two messages (SATELLITE ASSIGNMENT COMMAND and ASSIGNMENT COMPLETE/FAILURE) would be exchanged on the Air Interface and the entire procedure could be performed more rapidly. Fig. 5 shows the signaling flow for an Inter-FES Handover when the network does not know the MS position.

\section{Acknowledgments}

This work is partly based on the contributions of the University of Florence to the SAINT Project, coordinated by Alcatel Espace and to which University of Florence participated as an Associated Partner. We would like to thank all the partners of the SAINT project for the continuous exchanging of ideas in this two years work.

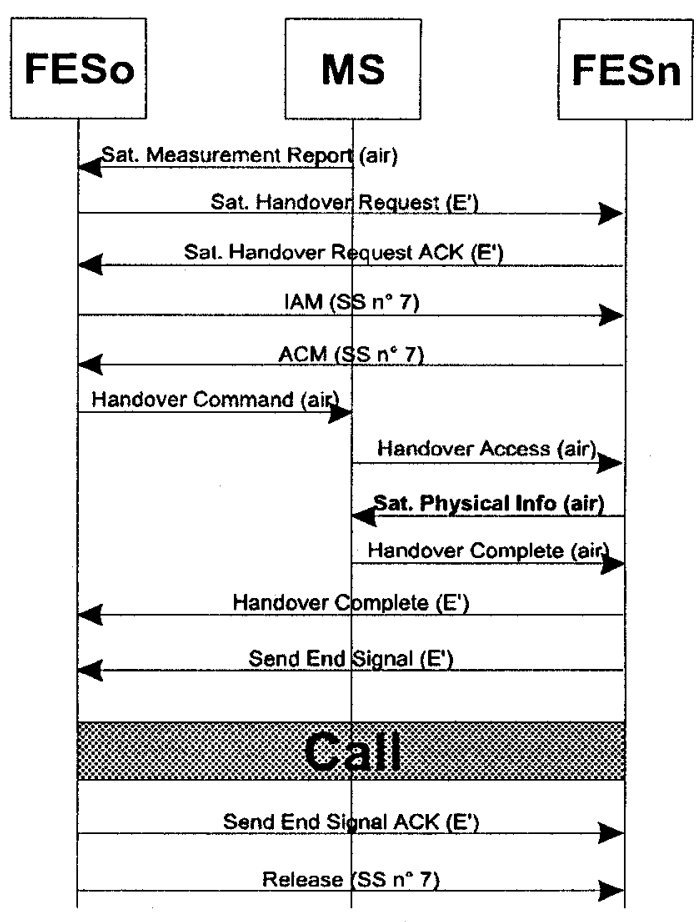

Figure 5 - Signaling flow for Inter-FES Handover

\section{References}

[1] SAINT Deliverable D16, "Interworking with other networks", Nov. 1995.

[2] E.Del Re, P.Iannucci, "The GSM Procedures in an Integrated Cellular/Satellite System", IEEE J. Select. Areas Comm., vol. 13, no. 2, pp. 421-430, Feb. 1995.

[3] J. Restrepo, G. Maral, "Coverage concepts for satellite constellations providing communications services to fixed and mobile users", Space Communications, Vol. 13, No. 2, 1995 , pp. 145-157.

[4] M.Dinis, J.Neves, R.Tafazolli, B.Evans, "A Proposed Mobility Management Technique for Resonant Mobile Satellite Constellations", COST 227 Meeting, Noordwijk, The Netherlands, 27-28 Jan. 1994, TD (94) 02.

[5] SAINT Deliverable D15, "Operations and procedures in integrated UMTS-satellite network", Nov. 1995.

[6] SAINT Deliverable D22, "Feasibility of migration from second generation systems to UMTS", Nov. 1995.

[7] C.Cullen, A.Sammut, R.Tafazolli, B.G.Evans, "Networking and Signalling Aspects of a Satellite Personal Communications Network", Proc. of the 1st European Workshop on Mobile/Personal Satcoms (EMPS'94), Frascati, Italy, 13-14 Oct., 1994, pp.202-222.

[8] M.Dinis, J.Neves, "Location Technique Implication in the Mobility Management Signalling for Satellite Mobile Networks Application", Proc. of the 2nd Joint COST 227/231 Workshop on Mobile and Personal Communications, Florence, Italy, 20-21 April, 1995, pp. 153-162. 